$\mathrm{kgAT})(+2.44 \mathrm{ml} / \mathrm{kg} / \mathrm{min}$ [CI 0.6,4.2], $\mathrm{p}=0.009)$, time to AT $(+115 \mathrm{~s}$ [CI 54.3,175.9], $\mathrm{p}<0.001)$ and exercise time (max ET) $(+108 \mathrm{~s}$ [CI 33.7,182.2], p=0.005). The exercise group also demonstrated greater reduction in systolic BP $(-7.3$ mmHg [CI -11.7,-2.8], p=0.002), BMI $\left(-0.8 \mathrm{~kg} / \mathrm{m}^{2}\right.$ [CI-1.1,$0.4], \quad \mathrm{p}<0.001)$, anxiety $(-2.6 \quad[\mathrm{CI}-3.6,-1.6], \quad \mathrm{p} \leq 0.001)$ and depression (-1.1 [CI -2.0,-0.2], $\mathrm{p}=0.015)$ scores. At T6m patient reported exercise adherence was comparable to baseline PA, in 33/34 of the exercise group attending for follow up. Most exercise gains dissipated with the exception of time to $\operatorname{AT}(p=0.002), \max \operatorname{ET}(p=0.003), \operatorname{VO} 2 / \mathrm{kgAT} \quad(p=0.04)$ and anxiety score $(\mathrm{p}<0.001)$. There were no sustained episodes of atrial or ventricular arrhythmias. The incidence of NSVT did not differ between time points $(p=0.09)$.

Conclusion A 12-week HIT programme in young patients with HCM offers considerable gains in fitness and psychological outcomes, with no increase in arrhythmic burden. Further research is still required to assess the long-term safety of high intensity exercise in the HCM population. At T6m exercise levels as well as most physiological adaptations and health benefits returned to baseline, as seen in other studies when formal participation in an exercise programme comes to an end. This highlights the importance of the implementation of strategies to encourage ongoing engagement in PA. Potential solutions include identification of barriers to exercise, as well as adoption of novel tele-rehabilitation approaches.

\section{IMPAIRED MYOCARDIAL ENERGETICS AS THE BASIS FOR EXERCISE-INDUCED PULMONARY CONGESTION IN HEART FAILURE WITH PRESERVED EJECTION FRACTION}

${ }^{1}$ Matthew K Burrage*, ${ }^{1}$ Moritz Hundertmark, ${ }^{2}$ Jack J Miller, ${ }^{1}$ Andrew JM Lewis*, ${ }^{1}$ Oliver J Rider*. 'University of Oxford Centre for Clinical Magnetic Resonance Research, Radcliffe Department of Medicine, University of Oxford, OX3 9DU, UK; ${ }^{2}$ Department of Physics, Clarendon Laboratory, University of Oxford, OX1 3PU, UK; * contributed equally as senior authors

\subsection{6/heartjnl-2021-BCS.237}

Background Abnormal cardiac mitochondrial function and energetics are implicated in the pathogenesis of heart failure with preserved ejection fraction (HFpEF). Transient pulmonary congestion during exercise is emerging as an important determinant of reduced exercise capacity and symptoms in HFpEF. We sought to determine if an abnormal cardiac energetic state underpins this process.

Methods We recruited 42 patients across the spectrum of diastolic dysfunction and HFpEF (healthy controls $n=10$, type 2 diabetes (T2DM) $n=9$, HFpEF $n=14$, and severe diastolic dysfunction due to cardiac amyloidosis $n=9$ ). Cardiac energetics were measured using phosphorus spectroscopy to define the myocardial phosphocreatine to adenosine triphosphate ratio (PCr/ATP). Cardiac function was assessed by cardiovascular magnetic resonance (CMR) cine imaging and echocardiography, and pulmonary congestion using MR proton density mapping. Studies were performed at rest and during submaximal exercise using an MRI-ergometer.

Results Paralleling the stepwise decline in diastolic function across the groups $(\mathrm{E} / \mathrm{e}$ ' ratio $\mathrm{p}<0.001)$ was an increase in NTpro BNP $(\mathrm{p}<0.001)$ and a reduction in $\mathrm{PCr} / \mathrm{ATP}$ (control 2.00 [1.86, 2.15], T2DM 1.71 [1.61, 1.91], HFpEF 1.66 $[1.44,1.89]$, cardiac amyloidosis $1.30[1.16,1.53], \mathrm{p}<0.001)$. During 20W exercise, reduced left ventricular (LV) diastolic filling rate $(r=0.41, p=0.008)$, left atrial (LA) dilatation $(r=-$
0.35, $\mathrm{p}=0.03)$, reduced right ventricular (RV) contractile reserve (RV ejection fraction change $r=0.46, p=0.003$ ), reduced right ventricular-pulmonary arterial (RV-PA) coupling $(\mathrm{r}=0.36, \mathrm{p}=0.02)$ and right atrial dilatation $(\mathrm{r}=-0.68$, $\mathrm{p}<0.001)$ were all linked to this reduction in PCr/ATP. Along with these changes, proton-density mapping revealed transient pulmonary congestion in patients with HFpEF $(+4.4 \%[0.5$, 6.4]) and cardiac amyloidosis (+6.4\% [3.3, 10]), which was not seen in healthy controls $(0.25 \%[-1.8,3.1])$ or T2DM without HFpEF $(0.8 \%$ [-1.7, 1.9]). Importantly, the development of exercise-induced pulmonary congestion was associated with reduced PCr/ATP $(r=-0.36, p=0.02)$.

Conclusions A gradient of myocardial energetic deficit exists across the spectrum of HFpEF. This energetic deficit is related to markedly abnormal exercise responses in all four cardiac chambers, which leads to detectable pulmonary congestion. The findings support an energetic basis for transient exerciseinduced pulmonary congestion in HFpEF.

\section{RADIOTRANSCRIPTOMIC ANALYSIS OF PERIVASCULAR ADIPOSE TISSUE QUANTIFIES VASCULAR INFLAMMATION IN COVID-19 FROM ROUTINE CT ANGIOGRAMS: STRATIFICATION OF "NEW UK VARIANT" INFECTION AND PREDICTION OF IN- HOSPITAL OUTCOMES}

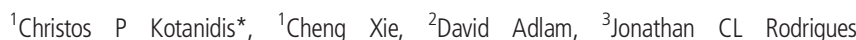
${ }^{1,4}$ Muhammad Siddique, ${ }^{5}$ Helen Lockstone, ${ }^{1,6}$ Sheena Thomas, ${ }^{1}$ Rafail Kotronias, ${ }^{1,7}$ Evangelos K Oikonomou, ${ }^{8}$ Sheila F Lumley, ${ }^{1}$ Stefan Neubauer, ${ }^{9}$ David E Newby, ${ }^{1,10}$ Keith M Channon, ${ }^{11}$ John Deanfield, ${ }^{1,6}$ Charalambos Antoniades. 'Division of Cardiovascular Medicine, Radcliffe Department of Medicine, University of Oxford, UK; ${ }^{2}$ Department of Cardiovascular Sciences and NIHR Leicester Biomedical Research Centre, University of Leicester, UK; ${ }^{3}$ Department of Radiology, Royal United Hospitals Bath NHS Foundation Trust, UK; ${ }^{4}$ Caristo Diagnostics, Oxford, UK; ${ }^{5}$ Wellcome Trust Centre for Human Genetics, University of Oxford, UK; ${ }^{6}$ Acute Vascular Imaging Centre, NIHR Biomedical Research Centre, John Radcliffe Hospital, Oxford University Hospitals NHS Foundation Trust and University of Oxford, UK; ' Department of Internal Medicine, Yale-New Haven Hospital, Yale School of Medicine, New Haven, USA; ${ }^{8}$ Nuffield Department of Medicine, University of Oxford, UK; ${ }^{9}$ British Heart Foundation Centre for Cardiovascular Science, University of Edinburgh, UK; ${ }^{10}$ British Heart Foundation-National Institute of Health Research Cardiovascular Partnership; ${ }^{11}$ Institute of Cardiovascular Sciences, University College London, London, UK

\subsection{6/heartinl-2021-BCS.238}

Background Evidence suggests that adverse outcomes in COVID-19 may be driven by a cytokine-induced vascular inflammatory response, caused by SARS-CoV-2 (severe acute respiratory syndrome coronavirus 2).

Aim We aimed to develop a non-invasive method for quantifying cytokine-driven vascular inflammation in patients with acute COVID-19 infection that could allow risk stratification. Methods We developed a platform for rapid development of novel imaging biomarkers of vascular inflammation, by applying quantitative radiotranscriptomics to images from standard Computed Tomography Angiography (CTA). We used this platform to train a radiotranscriptomic signature (C19-RS) from the perivascular space around the aorta and the internal mammary artery, visualized in routine chest CTAs, to best describe cytokine-driven vascular inflammation, defined using transcriptomic profiles from RNA sequencing data from human arterial biopsies. This signature was tested externally in 435 clinically indicated CT pulmonary angiograms (CTPAs) from patients with or without COVID-19 from 3 different geographical regions. 
Results COVID-19 patients were characterised by significantly higher C19-RS values (adjusted odds ratio of 2.97 [95\%CI: 1.43-6.27], $\mathrm{p}=0.004$ ), while patients infected with the new B.1.1.7 variant ("UK variant") were also found to have higher C19-RS values compared to those with the original variant, evidence suggestive of higher degrees of vascular inflammation. C19-RS had prognostic value for in-hospital mortality in COVID-19, with hazard ratios of 3.31 ([95\%CI: 1.49-7.33], $\mathrm{p}=0.003)$ and 2.58 ([95\%CI: 1.10-6.05], $\mathrm{p}=0.028)$ in two external testing cohorts respectively, after correction for clinical factors and biochemical biomarkers of inflammation (WBC, CRP) and myocardial injury (troponin). Importantly, the corrected $\mathrm{HR}$ for in-hospital mortality was 8.24[95\%CI: 2.1631.36], $\mathrm{P}=0.002$ for those who received no treatment with dexamethasone, but only 2.27[95\%CI: 0.69-7.55], $\mathrm{p}=0.18$ in those who received dexamethasone after the test, suggesting that anti-inflammatory treatment may be modifying the natural history of COVID-19 infection by improving outcomes specifically in those patients with high vascular inflammation.

Conclusions Our study introduces a new radiotranscriptomic signature, C19-RS, extracted from routine CTPAs, trained to detect cytokine-driven arterial inflammation, and demonstrates that vascular inflammation determined in this way has prognostic value in patients with COVID-19. The "UK variant" leads to higher vascular inflammation measured in this way, and the risk associated with COVID-19 arteritis is modifiable by dexamethasone.

\section{\begin{tabular}{|l|l}
\hline E & ABNORMAL RESTING ECG T-WAVE MORPHOLOGY
\end{tabular} PREDICTS VENTRICULAR ARRHYTHMIC RISK IN A LARGE "LOW-RISK" COHORT}

Julia Ramírez.

\subsection{6/heartjnl-2021-BCS.239}

Introduction Early identification of individuals in the general population at high sudden cardiac death (SCD) risk remains a major challenge. The T-wave morphology reflects ventricular repolarization dispersion, one of the main contributors to ventricular arrhythmias (VAs) leading to SCD. Deviations of traditional T-wave indices, like the corrected QT (QTc) interval or the T-peak-to-T-end (Tpe) interval, from standard thresholds indicate increased risk. However, there is currently no index quantifying overall T-wave morphological deviations from a normal reference, even though it could be a more robust VA risk marker. The aim of this study is to test the predictive value of a novel index quantifying T-wave morphology deviations from a normal reference.

Methods sex-, heart rate-, and electrocardiogram (ECG) lead-specific T-wave morphology references were extracted from standard 10-s 12 leads ECGs from 23,962 participants in the UK Biobank imaging study. We, then, calculated the difference between the T-wave morphologies from 51,794 independent participants without a previous history of cardiovascular events in the UK Biobank and their corresponding sex-, heart rate- and ECG lead-specific T-wave morphology using time-warping metrics. In particular, we derived the TMT index, quantifying T-wave morphology deviations in time, and its predictive power was compared to that of traditional risk factors and standard T-wave indices for two endpoints. The primary endpoint was VA mortality or hospitalizations for VA reasons. The secondary endpoint was major adverse cardiovascular events, defined as either hospitalization or death due to myocardial infarction heart failure or ventricular arrhythmia. The median follow-up time was 122 months.

Results A total of $220(0.4 \%)$ individuals reached the primary endpoint, and 4,786 (9.2\%) was significantly higher in individuals meeting the primary endpoint compared to controls (median 1.8 versus $1.6, P=0.003$ ). Multivariable Cox analysis revealed that TMT was significantly associated with the primary endpoint (hazard ratio (HR) 1.13, 95\% confidence interval $[\mathrm{CI}]$ of $1.04-1.25, P=0.007$ ) independently of standard cardiovascular risk factors, as well as resting heart rate, T-wave inversions, resting Tpe interval and QTc interval. TMT was also significantly associated with the secondary endpoint (HR 1.06, 95\% CI of $1.03-1.08, P<1 \times 10^{-3}$ ).

Conclusion This study shows that an abnormal T-wave morphology at rest is associated with VA risk in a cohort from the general population without previous cardiovascular conditions independent of standard cardiovascular risk factors and a better predictor than traditional ECG risk markers. Our findings support the hypothesis that the overall morphology of the T-wave is able to capture increased dispersion of ventricular repolarization than traditional T-wave indices, identifying a high-risk substrate for malignant VAs that could lead to SCD. 